\title{
NAVIGATION AND MOTION CONTROL SYSTEMS OF THE AUTONOMOUS UNDERWATER VEHICLE
}

\author{
Dmitry Antonov ${ }^{1}$ \\ oxface@yandex.ru \\ Leonid Kolganov ${ }^{1}$ \\ leon.kolg@gmail.com \\ Aleksey Savkin \\ yolka583@yandex.ru \\ Egor Chekhov ${ }^{1}$ \\ egorchekhov@icloud.com \\ Maxim Ryabinkin \\ max230658@gmail.com \\ ${ }^{1}$ Department "NIO-305" \\ Moscow Aviation Institute (National Research University) \\ 4 Volokolamskoe highway, Moscow, Russia, 125993
}

\begin{abstract}
Autonomous underwater vehicles (AUVs) are widely used and have proven their effectiveness in tasks such as transportation safety, area monitoring and seafloor mapping. When developing AUV's navigation and control systems, the engineers have to ensure the required levels of accuracy and reliability for solving navigation and motion control tasks in autonomous underwater operation under restrictions on the overall dimensions and power consumption of the AUV. The main purpose of this paper is to present preliminary results of AUV navigation and motion control systems development.

The AUV's navigation system is built around strapdown inertial navigation system (SINS) designed specifically for this AUV. When surfaced, position and angular SINS correction is performed using data from dual-antenna GNSS receiver and doppler velocity $\log (\mathrm{DVL})$. When underwater, SINS position and velocity correction is performed using acoustic navigation system (ANS) and DVL data.

AUV's control system provides manual and automatic control. Manual control is carried out in real-time by operator via fiber-optic cable using a joystick. Automatic control allows AUV to move independently along a specified trajectory at a given depth and speed. The AUV also has a collision avoidance system that utilizes readings from a forward-facing acoustic rangefinder to estimate time before impact based on AUV's analytic model. If possible collision is detected, information is transmitted to the control system so that a further appropriate action can be taken.

Computer simulation utilizing the analytic AUV model was used in order to check the performance characteristics of the designed control and navigation algorithms. After confirming the operability of the developed algorithms, preliminary tests of the AUV were carried out. During the tests, AUV's on-board equipment and navigation system readings were recorded and compared to the readings of the reference system, which was also installed on the AUV. During the tests, the dynamic characteristics of the AUV were evaluated. AUV's characteristics obtained during simulation and testing will be used as a reference during future development.
\end{abstract}

Keywords: autonomous underwater vehicle, control system, inertial navigation system, Kalman filter, navigation.

DOI: $10.21303 / 2461-4262.2020 .001361$

\section{Introduction}

Autonomous underwater vehicles (AUVs) are widely used and have proven their effectiveness in tasks such as transportation safety, area monitoring and seafloor mapping. According to the Russian Federation state standard GOST R 56960-2016, AUVs are classified as it is shown in the Fig. 1 [1].

The most popular AUVs in civil applications weight around $100 \mathrm{~kg}$. These AUVs are actively used by research groups and rescue services due to relatively compact size and low weight, 
facilitating fast deployment by a small crew. Most of these AUVs have a modular structure [2-5], which allows them to carry a wide variety of equipment.

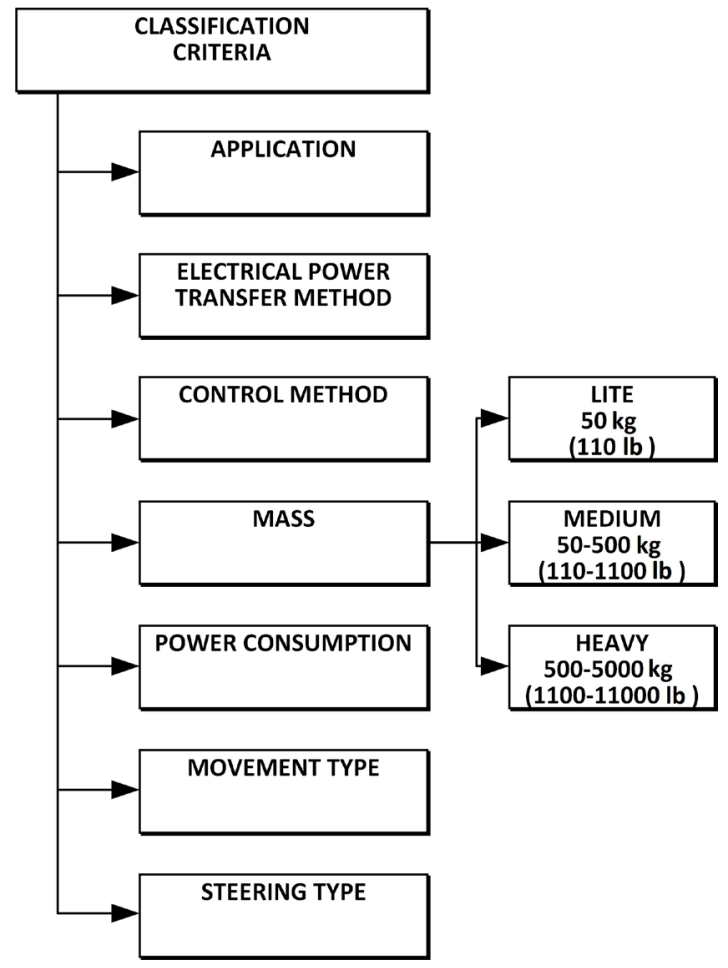

Fig. 1. AUV classification based on mass

Operation of communication, control and navigation systems can be divided into two modes: surface and underwater. The main operating mode is underwater. In this mode, AUV can be controlled by an operator using a fiber optic cable, or it can carry out a mission autonomously according to the pre-programmed task. A cable system removes restrictions on energy consumption and assures a stable high-speed data connection for real-time manual or semi-automatic control of the AUV. However, any cable system has several drawbacks:

- underwater currents apply a strong force to the cable, which pulls the AUV; this affects AUV's maneuverability and greatly reduces its ability to stabilize;

- a cable system limits AUV's effective range.

Because of the mentioned disadvantages, AUV described in this paper is usually operated without cable. When below water surface, the use of radio communications (Wi-Fi, satellite) is not possible. In practice, the acoustic modem is the only way of communication with the submersed AUV.

When developing AUV's navigation and control software, the engineers have to ensure the required levels of accuracy and reliability taking into account an overall size and power consumption restrictions. For example, in [2] a general purpose AUV is equipped with variable buoyancy module which allows for power efficient operation making it a hybrid between AUV and a glider. Smaller and power efficient AUVs such as in [2] are easier to manufacture and maintain, but a lot of specialized, research and general purpose equipment require a larger battery. A heavier AUV is described in [3], which is equipped with sonar for obstacle avoidance as well as water quality analysis device, but can only be autonomous for 3 hours. AUVs described in [4, 5] are similar in terms of equipment, but also feature a side scan sonar and can be autonomous for a longer time. An AUV described in this paper was designed with the goal of having a low enough power consumption to be able to sustain a prolonged period of time underwater ( $>10$ hours) and also be able to carry power demanding loads. The research objective was to design and evaluate navigation and motion control systems for the developed AUV. The paper presents preliminary results of AUV navigation and motion control systems development. 


\section{A brief description of the developed AUV}

AUV described in this paper has characteristics shown in the Table 1.

Table 1

AUV characteristics

\begin{tabular}{|c|c|}
\hline Parameter & Value \\
\hline Mass & From 100 to $125 \mathrm{~kg}(220$ to $275 \mathrm{lb})$ \\
\hline Water displacement & From 900 to 9501 (200 to $210 \mathrm{gal})$ \\
\hline External diameter & $205 \mathrm{~mm}$ (8 in) \\
\hline Cruising speed & $2.5 \mathrm{~m} / \mathrm{sec}(4.8 \mathrm{knots})$ \\
\hline Max depth & $300 \mathrm{~m}(985 \mathrm{ft})$ \\
\hline
\end{tabular}

The developed AUV can be classified as it is shown in the Table 2.

Table 2

AUV classification

\section{Classification criteria}

Application

Electrical power transfer method

Control method

Mass

Power consumption

Movement type

Steering type

\section{Class}

Multipurpose

Autonomous

Automatic

Medium

Small

Self-propelled

Active

The hardware structure of the AUV's on-board equipment is shown in the Fig. 2.

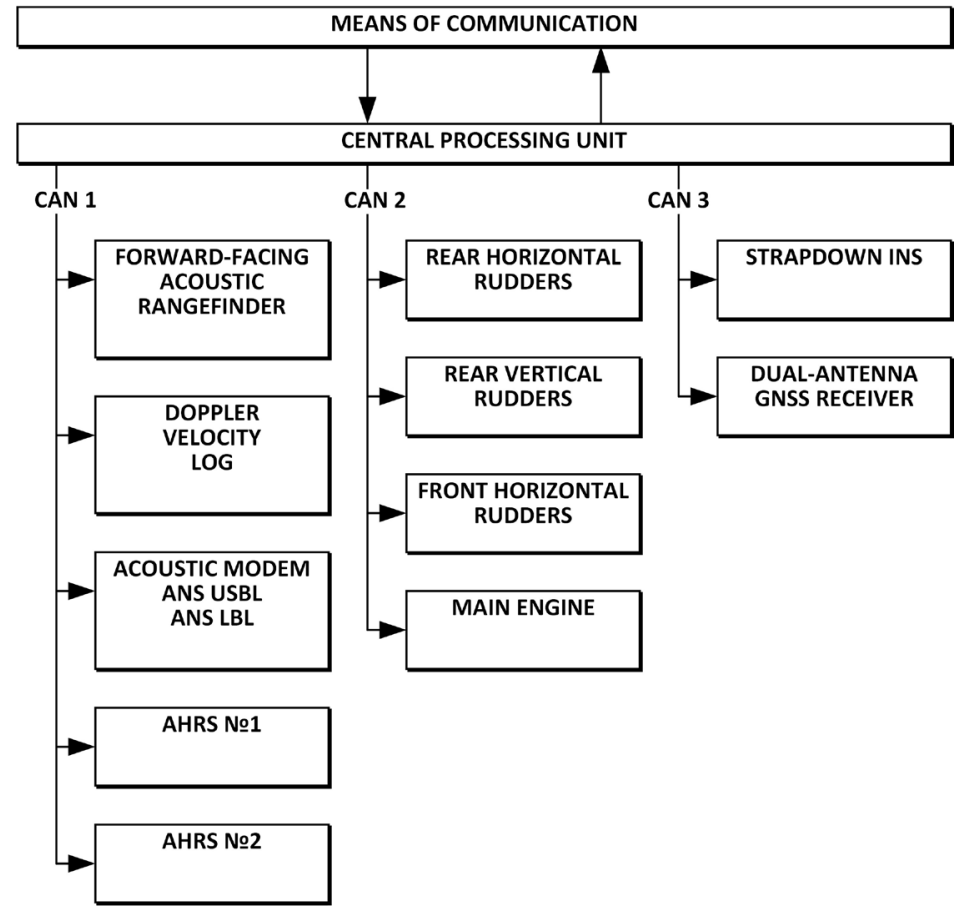

Fig. 2. The developed AUV's on-board equipment structure: ANS LBL is the acoustic navigation system long baseline; ANS USBL is the acoustic navigation system ultrashort baseline;

INS is the inertial navigation system; GNSS is the global navigation satellite system;

AHRS is the attitude and heading reference system 
The allocation of the equipment inside the AUV is shown in the Fig. 3. The developed AUV has a modular structure, which allows for the various configurations in accordance with the presented task.

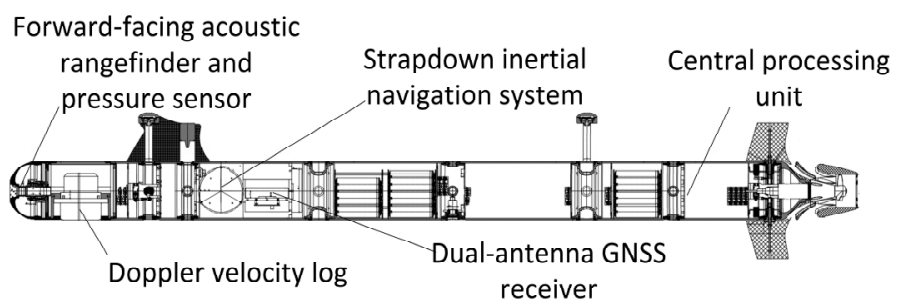

Fig. 3. The developed AUV's internal structure

Data exchange between the equipment and a central processing unit is carried out using CAN bus via special interface modules. Interface modules convert data and voltage from the on-board network to the desired format and levels, matching the equipment specification. Interface modules also monitor power consumption and protect equipment from critical operating conditions.

\section{Navigation system}

The AUV's navigation system is built around strapdown inertial navigation system (SINS) designed specifically for this AUV. Navigation system algorithmic structure is shown in the Fig. 4. Inertial measurement unit (IMU) utilizes navigation grade gyroscopes and accelerometers, which are designed and manufactured in Russian Federation. SINS unit is shown in the Fig. 5.

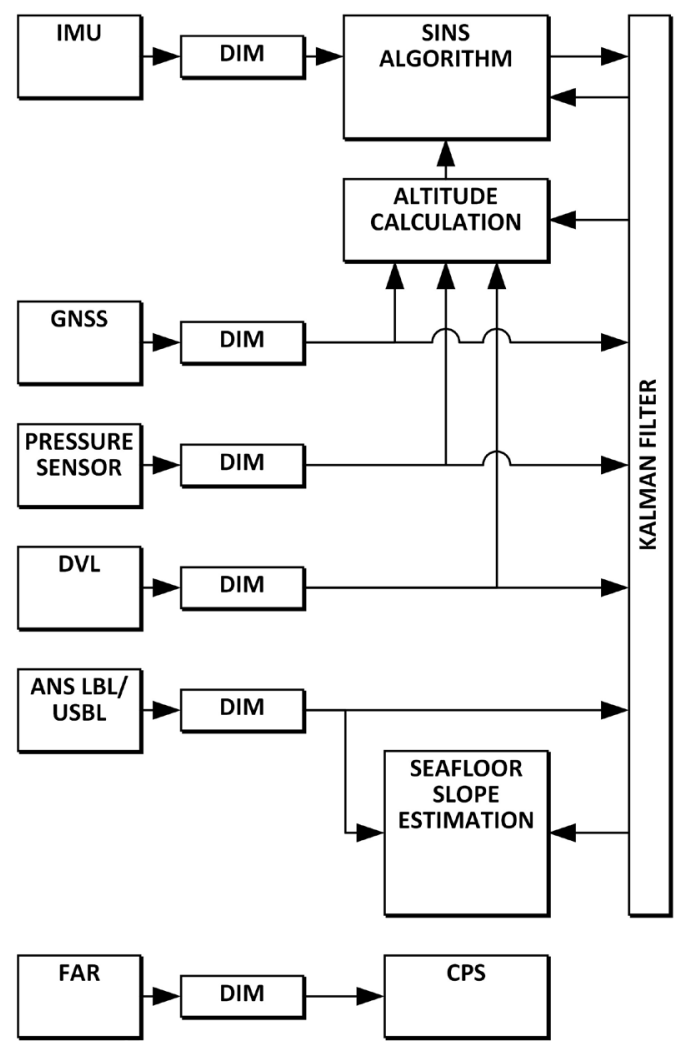

Fig. 4. The AUV's navigation system algorithmic structure: IMU is the inertial measurement unit; DIM is the data interpolation module; SINS is the strapdown inertial navigation system;

GNSS is the global navigation satellite system; DVL is the Doppler velocity lag;

ANS LBL/USBL is the acoustic navigation system long baseline/ultrashort baseline;

FAR is the forward-facing acoustic rangefinder; CPS is the collision prevention system 


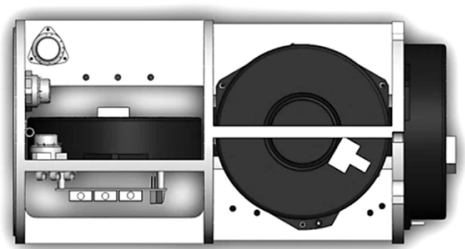

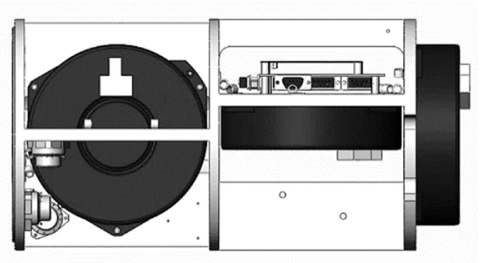

$b$

Fig. 5. The AUV's strapdown inertial navigation system: $a$ - right view; $b$ - top view

The AUV's vertical position is calculated based on the data provided by global navigation satellite system (GNSS), by pressure sensor and by IMU using standard Kalman filter.

INS initial alignment can be achieved in two ways:

- autonomous, using only IMU data;

- aided, using phase measurements from dual-antenna GNSS receiver, which are the part of attitude and heading reference systems, which are located in front and rear parts of the AUV.

When surfaced, position and angular SINS correction is performed using data from dual-antenna GNSS receiver [5] and Doppler velocity log (DVL). When underwater, SINS position and velocity correction is performed using acoustic navigation system (ANS) and DVL data. Let's also currently study the possibility of using side-scan sonar to aid SINS when moving underwater. SINS correction is performed using algorithms based on Kalman filter by triple redundant central processing unit, shown in the Fig. 6.

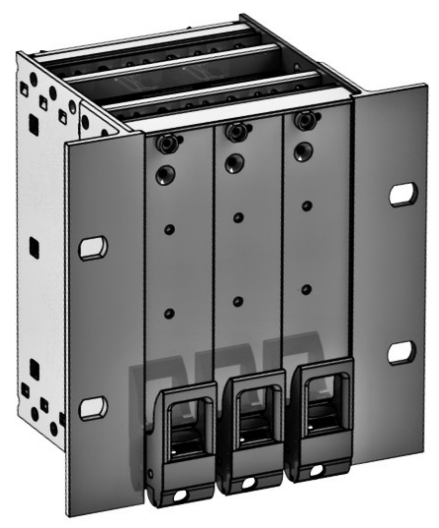

Fig. 6. The triple redundant central processing unit

Kalman filter is implemented and modified in a way which allows for the separation of estimation, correction and control cycles, thus even in the absence of correction data, accuracy of calculated parameters can be estimated. Filter state vector consists of SINS errors and has a following form:

$$
\overrightarrow{\mathrm{X}}^{T}=\left[\begin{array}{c}
x_{1}, x_{2}, x_{3}, x_{4}, \alpha, \beta, \gamma \\
\Delta \Omega_{X}, \Delta \Omega_{Y}, \Delta \Omega_{Z}, \Delta K_{\Omega_{X}}, \Delta K_{\Omega_{Y}}, \Delta K_{\Omega_{Z}}, \\
\Delta n_{X}, \Delta n_{Y}, \Delta n_{Z}, \Delta K_{n_{X}}, \Delta K_{n_{Y}}, \Delta K_{n_{Z}}
\end{array}\right],
$$

where $x_{1}, x_{2}$ are the position errors; $x_{3}, x_{4}$ are the velocity errors; $\alpha, \beta, \gamma$ are the heading and attitude errors; $\Delta \Omega_{X}, \Delta \Omega_{Y}, \Delta \Omega_{Z}$ are the gyroscope zero offset errors; $\Delta K_{\Omega_{X}}, \Delta K_{\Omega_{Y}}, \Delta K_{\Omega_{Z}}$ are the gyroscope scale factor errors; $\Delta n_{X}, \Delta n_{Y}, \Delta n_{Z}$ are the accelerometer zero offset errors; $\Delta K_{n_{X}}, \Delta K_{n_{Y}}, \Delta K_{n_{Z}}$ are the accelerometer scale factor errors.

All measurements from sensors and on-board equipment are synchronized to a common time scale with a resolution of $1 \mu \mathrm{s}$. Synchronization is performed via CAN bus. During synchronization, delays in data transmission lines, as well as drifts, and drift rates of local clock generators are estimated. The accuracy of synchronization according to the internal tests achieves the level of 
$1 \mathrm{~ms}$ (3 STD). Obtained measurements are then realigned and interpolated to a common time scale and passed to navigation and control algorithms (Fig. 4).

To study the properties of the developed navigation algorithms, computer simulation and field testing were carried out.

Computer simulation was performed using experimental data, obtained by the test vehicle [6] with reference navigation system (SPAN-SE by Novatel) installed. Simulations of autonomous (Fig. 7) and GNSS aided (Fig. 8) modes using the experimental data were performed.

INS true heading error (autonomous)

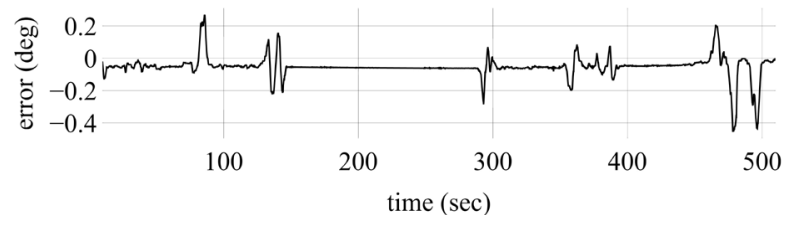

$a$

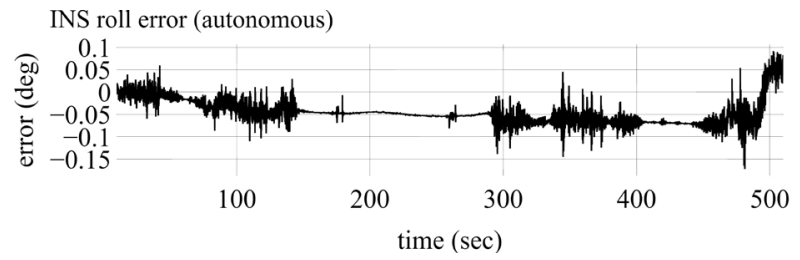

$c$
INS pitch error (autonomous)

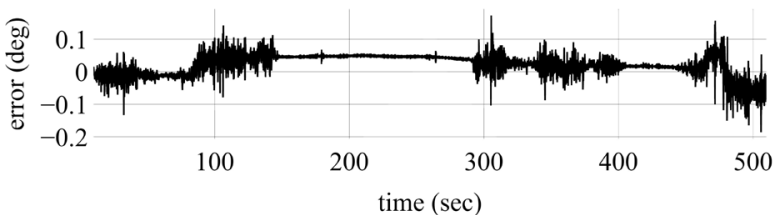

$b$

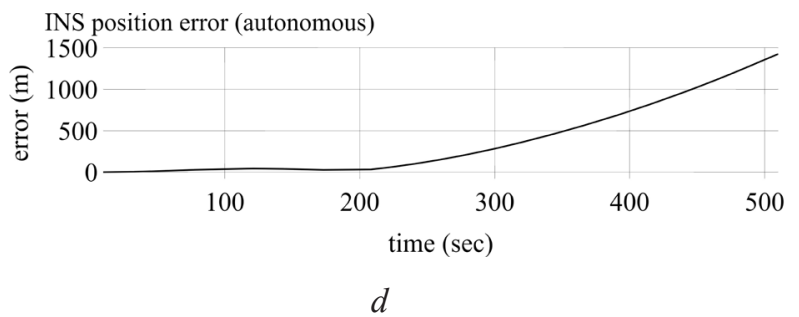

Fig. 7. Attitude and heading errors during autonomous INS operation (navigation system simulation using experimental IMU and GNSS data): $a$ - INS true heading error (aided);

$b$ - INS pitch error (aided); $c$ - INS roll error (aided); $d$ - INS position error (aided)

The presence of a high-frequency component in the angle errors originates in vibrations caused by car movement. It should be noted, that in the AUV experiments, these vibrations are of a much smaller intensity or negligible. The AUV's trajectory calculated by reference system based on the experimental data is shown in the Fig. 9.

INS true heading error (aided)

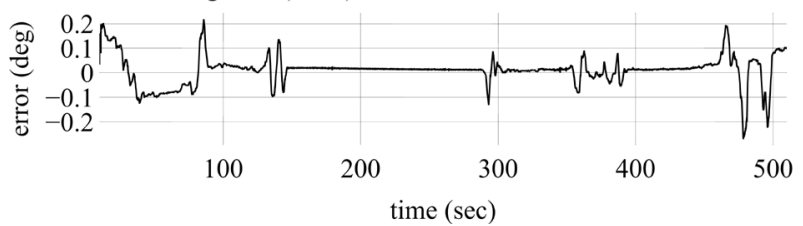

$a$

INS roll error (aided)

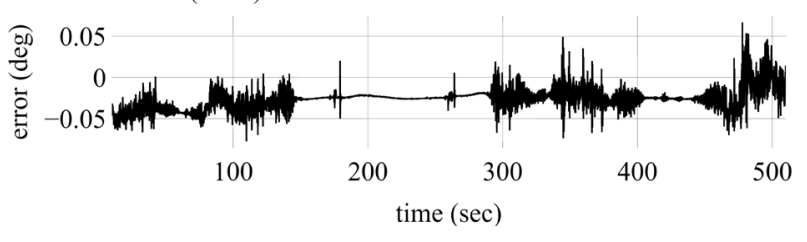

c
INS pitch error (aided)
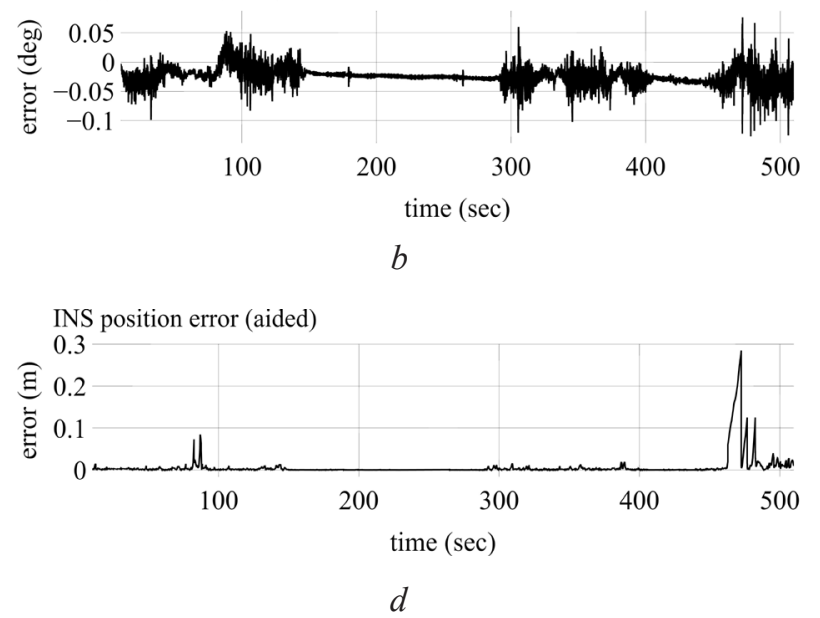

Fig. 8. The attitude and heading errors during GNSS aided INS operation (navigation system simulation using experimental IMU and GNSS data):

$a$ - INS true heading error; $b$ - INS pitch error; $c$ - INS roll error; $d$ - INS position error

Fig. 10 shows one of the field test scenarios. The AUV successively sank and surfaced, moving along a given trajectory underwater. 
During the field tests, AUV's on-board equipment and navigation system readings were recorded and compared to the readings of the reference system (Phins by iXblue), which was also installed on the AUV. Measured and reference orientation angles (heading, pitch, roll) are shown in the Fig. 11. Red indicates the reference system readings, green indicates the designed system readings, black indicates the angles obtained through GNSS phase measurements.

As can be seen in Fig. 11, calculated angles are closely following angles calculated by the reference system. Leveled off sections of the black line correspond to the absence of GNSS solution while AUV was moving underwater.

Overall, the main advantages of developed navigation algorithms are:

- internal measurement synchronization to a common time scale using CAN bus with an estimated accuracy of $1 \mathrm{~ms}$ (3 STD);

- independent estimation and correction cycles in Kalman filter, which allow to predict INS accuracy during unavailability of GNSS and other correctors;

- custom triple redundant processing unit for high reliability.

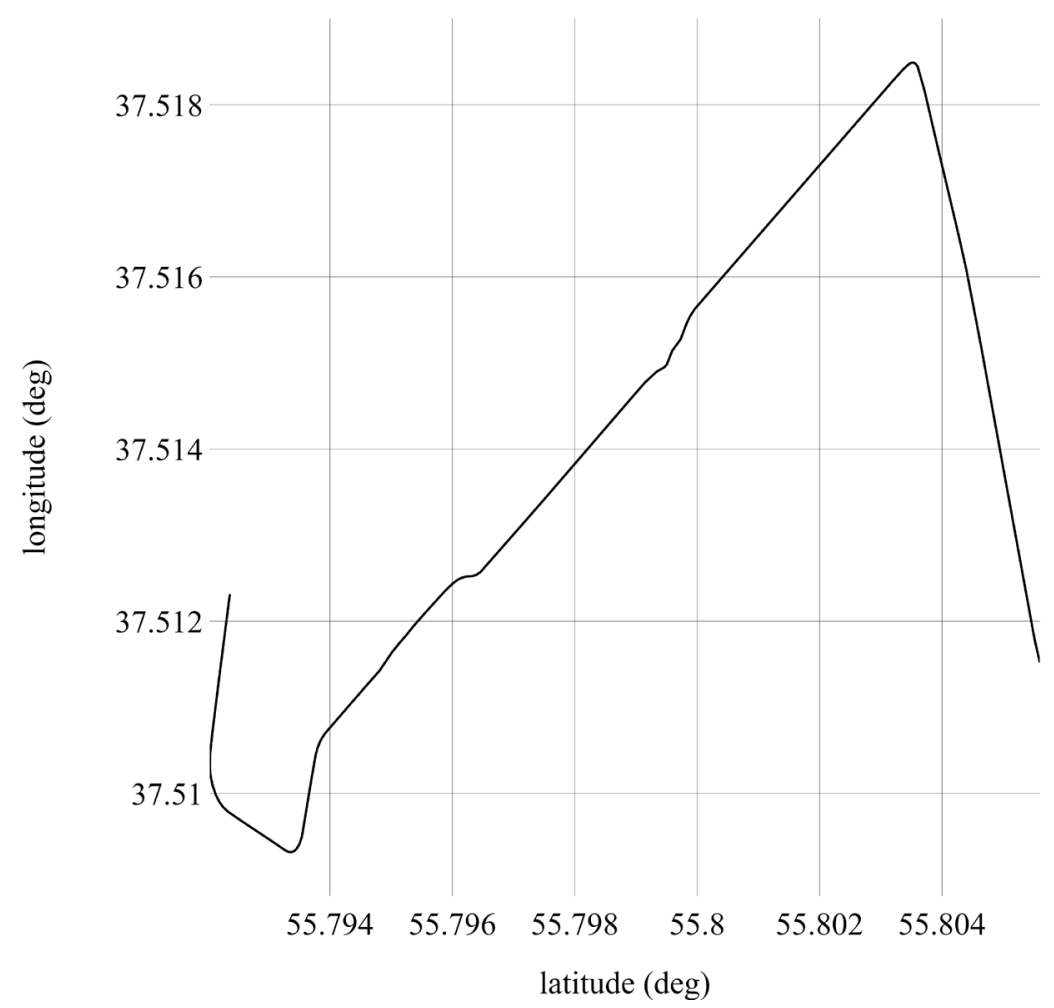

Fig. 9. Reference trajectory

(navigation system simulation utilizing experimental IMU and GNSS data)

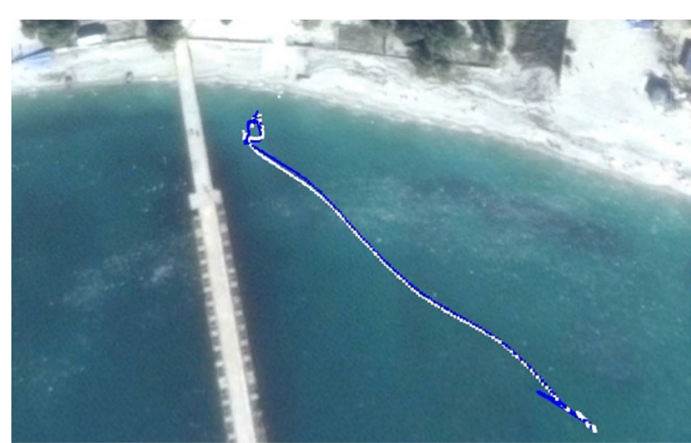

Fig. 10. The AUV's trajectories calculated by reference and designed systems (AUV prototype field testing with reference system installed) 


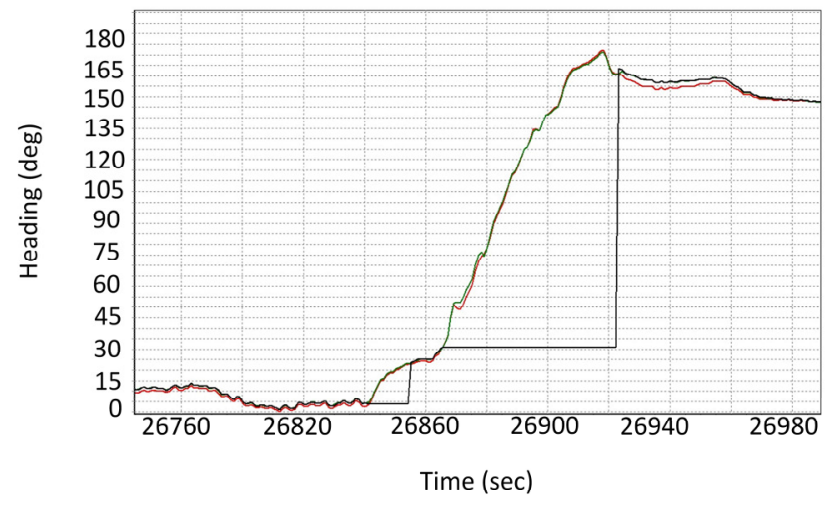

a

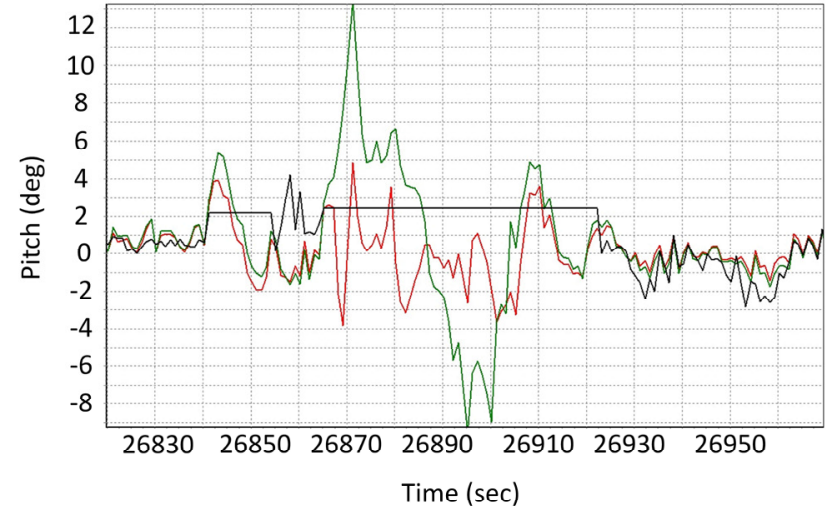

b

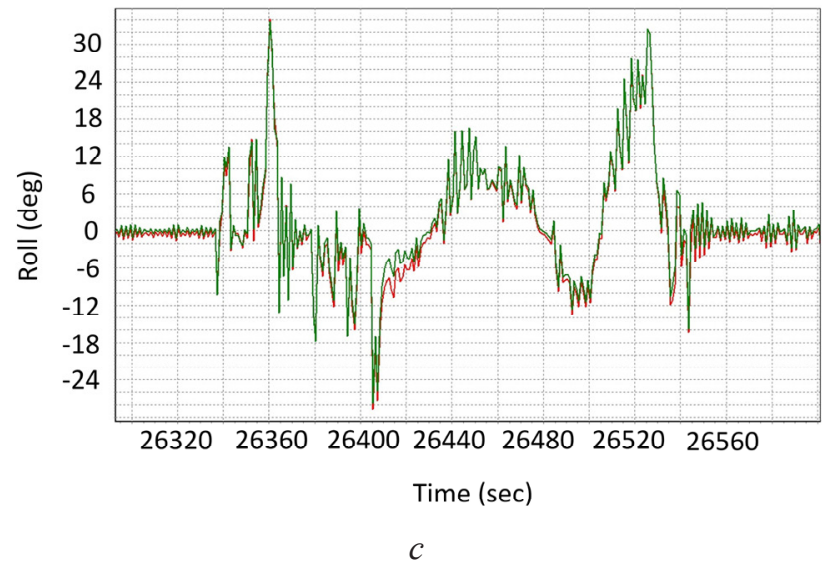

Fig. 11. Reference and measured attitude and heading (AUV prototype field testing with reference system installed): $a$ - heading; $b$-pitch; $c$ - roll

\section{Control system}

At the initial stage of control system development cycle, AUV's analytic model was developed. A generalized AUV model in vector form is:

$$
\begin{gathered}
\dot{\bar{Y}}=R \bar{X}, \\
M \dot{\bar{X}}=\bar{F}=\overline{F_{h}}+\overline{F_{d}}+\overline{F_{c}}+\overline{F_{m}},
\end{gathered}
$$

$\overline{F_{m}}$ are the forces and angular momentum associated with the center of mass and AUV buoyancy [7]. 
Parameterization of the model was carried out by the means of computer simulation [8]. During simulation, dependences of hydrodynamic coefficients on angle of attack, sideslip angle, and other parameters were obtained and linearized. These parameters allowed for the development of the AUV's analytic model, which takes into account the following:

- moving water mass effects on AUV's dynamics [9];

- structural elements drag;

- steering rudders effectiveness.

The resulting non-linear second order model consists of six equations and has series of cross-links between the equations, which allow for more accurate simulation of both simple and complex spatial AUV maneuvers, such as, for example, spiral movement.

Control system was developed using AUV's analytical model, which was reproduced in Simulink. AUV's control system provides manual and automatic control. Manual control is carried out in real-time by operator via fiber-optic cable using a joystick. Control system ensures that course, depth and speed specified by the operator are maintained. Automatic control allows for AUV's independent movement along a specified trajectory at given depth and speed.

Control system is divided into two blocks: stabilization unit and trajectory control unit. Stabilization unit ensures compliance of AUV's parameters with target parameters generated by trajectory control unit. Control system algorithmic structure is shown in Fig. 12.

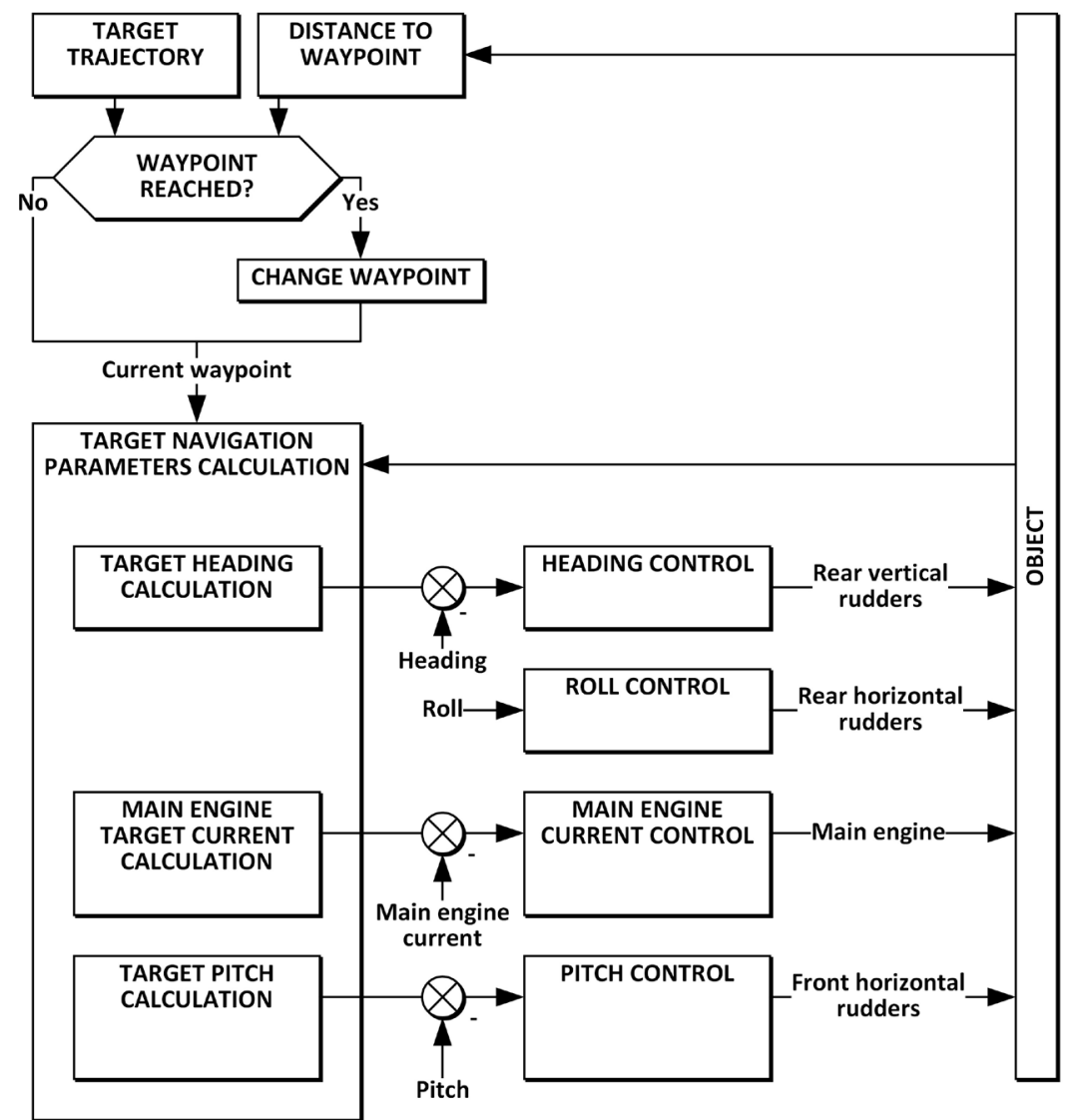

Fig. 12. Control system algorithmic structure

Low AUV's resonant frequency and three pairs of rudders made it possible to build a stabilization system comprised of three independent channels, which greatly reduced complexity of calculations.

The automatic control system supports various modes, such as changing depth with and without trim; roll stabilization; maintaining depth or distance from the bottom. 
The AUV also has a collision avoidance system that utilizes readings from a forward-facing acoustic rangefinder to estimate time before collision based on AUV's analytic model. If possible collision is detected, information is transmitted to the control system so that a further appropriate action can be taken.

Based on DVL bottom range data, the slope of the seafloor is approximated, which allows for AUV's movement at the fixed distance from the bottom.

In order to speed up the development process and simplify testing, all algorithms were implemented in Matlab/Simulink software with subsequent conversion to $\mathrm{C}$ language. This approach allowed to shorten build cycles. It also enabled instant verification of introduced changes through modelling based on a personal computer [10]. The use of the certified Simulink program code generators ensures that the generated program code matches the original model, thereby eliminating the human factor. Obtained code combined with microcontroller peripheral drivers was used to produce a single software package for the target platform.

Computer simulation utilizing the analytic AUV model was used in order to check the performance characteristics of the designed control system. During the simulation, the AUV has passed through predetermined waypoints at a given depth and speed. Waypoints are shown in Table 3.

Table 3

Trajectory waypoints

\begin{tabular}{ccccc}
\hline Way-point num. & Latitude & Longitude & Depth & Velocity \\
\hline 0 & $40.6920^{\circ} \mathrm{N}$ & $74.0452^{\circ} \mathrm{W}$ & $0 \mathrm{~m}$ & $0.0 \mathrm{~m} / \mathrm{sec}$ \\
& & & $0 \mathrm{ft}$ & $0.0 \mathrm{ml} / \mathrm{h}$ \\
1 & $40.6945^{\circ} \mathrm{N}$ & $74.0452^{\circ} \mathrm{W}$ & $5 \mathrm{~m}$ & $2.0 \mathrm{~m} / \mathrm{sec}$ \\
& & & $16 \mathrm{ft}$ & $3.9 \mathrm{knots}$ \\
2 & $40.6920^{\circ} \mathrm{N}$ & $74.0477^{\circ} \mathrm{W}$ & $33 \mathrm{ft}$ & $2.5 \mathrm{~m} / \mathrm{sec}$ \\
& & & $4.9 \mathrm{knots}$
\end{tabular}

The AUV's trajectory during simulation is shown in the Fig. 13.

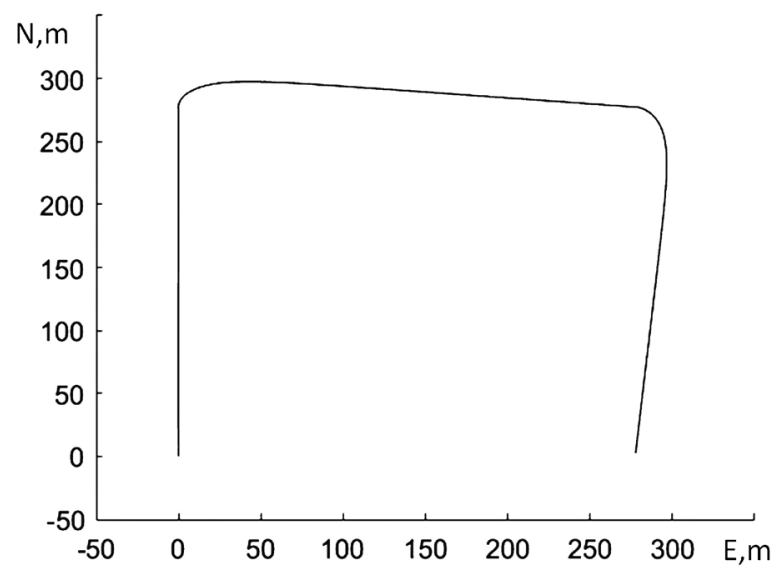

Fig. 13. The AUV's trajectory (control system simulation utilizing analytic AUV model)

In Fig. 13, the center of coordinates coincides with the starting point, $N$ axis is directed to the north, $E$ axis is directed to the east. The AUV's depth profile shown in the Fig. 14.

Orientation angles (heading, pitch, roll) are shown in Fig. 15.

Simulation results have proven operability of the implemented control algorithms. During the analysis of the simulation results, stable and unstable control modes were identified, as well as propulsion thrust necessary to achieve the estimated cruising speed. 


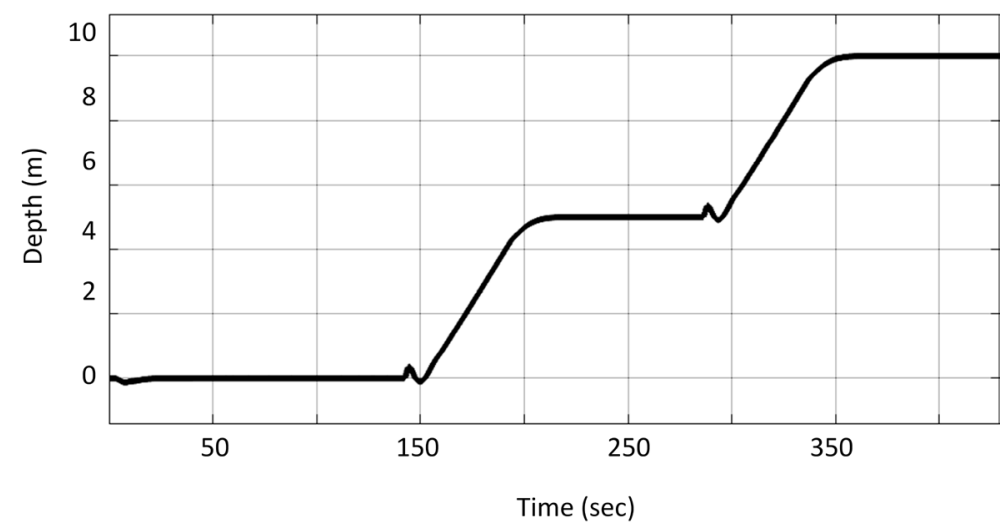

Fig. 14. The AUV's depth profile (control system simulation utilizing analytic AUV model)
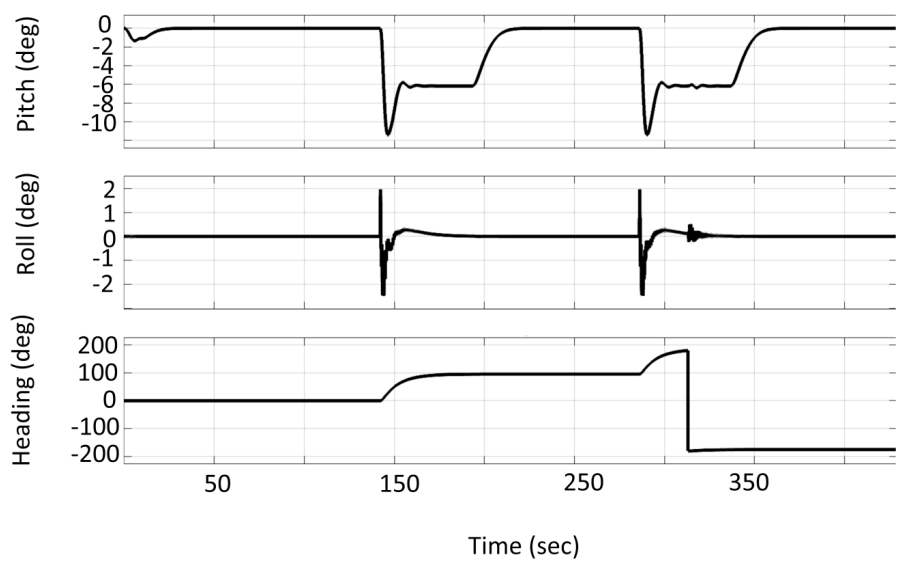

Fig. 15. Attitude and heading (control system simulation utilizing analytic AUV model)

After confirming the operability of control algorithms, preliminary tests of the AUV in an indoor pool were carried out (Fig. 16).

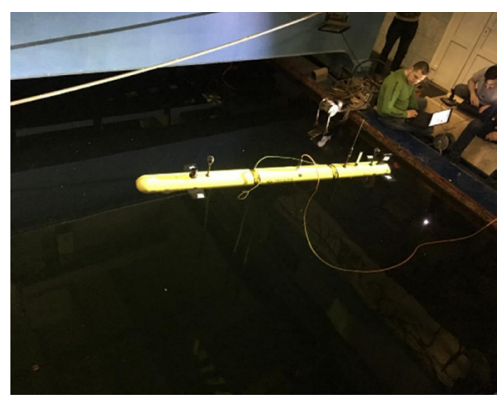

$a$

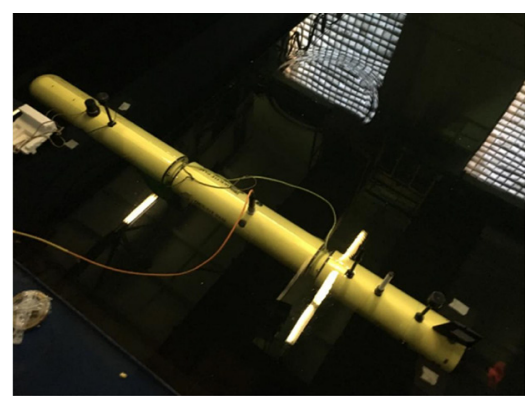

$b$

Fig. 16. The AUV pool testing: $a$ - The AUV side view; $b$ - The AUV top view

During the tests, the dynamic characteristics of the AUV were evaluated. Predicted (based on the analytic model) and measured AUV dynamic parameters are shown in the Table 4.

As can be seen in the Table 4, the first two parameters were approximately predicted. Other parameters couldn't be compared because the torque characteristics of the AUV's engine were obtained experimentally, and the rudder efficiency could not be obtained experimentally at the time.

In general, the main advantages of developed control algorithms are:

- control algorithms were designed and simulated based on the custom analytic model which was simple and accurate enough to run in real-time and closely approximate complex AUV movement; 
- AUV's control system features collision avoidance system, which predicts vehicle movement in real-time using the analytic model mentioned previously.

Table 4

Predicted and measured AUV dynamic parameters

\begin{tabular}{ccc}
\hline Parameter & Predicted value & Value obtained during test \\
\hline Roll resonant frequency & $1.5 \mathrm{~Hz}$ & $0.4 \mathrm{~Hz}$ \\
Horizontal turning radius & $\sim 25 \mathrm{~m}$ & $\sim 20 \mathrm{~m}$ \\
& $\sim 82 \mathrm{ft}$ & $\sim 65 \mathrm{ft}$ \\
Torque/current and torque/rpm propor- & & $0.0078\left[\frac{N}{A}\right]$ \\
tion coefficients & - & $0.0037\left[\frac{N}{R P M}\right]$ \\
Rudder efficiency coefficient & $1.551 \mathrm{~N} / \mathrm{m} \times \mathrm{rad}$ & -
\end{tabular}

\section{Discussion}

In this study, AUV navigation and motion control systems were designed and evaluated. Computer simulation using real data showed that in autonomous mode the angle errors are within 0.4 degrees (3 STD) margin after 10 minutes of movement (Fig. 7, $\boldsymbol{a}-\boldsymbol{c}$ ) and within 0.2 degrees (3 STD) margin when using the GNSS correction (Fig. 8, $\boldsymbol{a}-\boldsymbol{c}$ ). The position error does not exceed $0.3 \mathrm{~m}$ in aided mode (Fig. 8, $\boldsymbol{d}$ ), but in autonomous mode it starts to increase when approaching 4 min (Fig. 7, $\boldsymbol{d}$ ) mark which corresponds to the AUV making a sharp turn. Taking into account that these simulations were performed based on the real data provided by a tactical grade IMU mounted on a vehicle [6], it is possible to conclude that these results are indeed sufficient to prove the operability of the developed algorithms.

Field tests using onboard navigation grade IMU and a reference system Phins by iXblue refuted our concerns about the increase of the position error in autonomous mode shown by the computer simulation. Real-world tests proved that the developed AUV is reliably operated underwater, and the reference and onboard calculated trajectories closely match (Fig. 10).

Control system evaluation and tuning were performed using computer simulation based on the analytical model. Simulation showed that AUV successfully passed the predefined waypoints (Table 3) which can be seen from Fig. 13 without much overshoot (Fig. 13, 14). During field testing PID parameters were further corrected.

Over the course of the AUV development new hardware and software solutions were introduced:

- internal measurement synchronization to a common time scale using CAN bus with an estimated accuracy of $1 \mathrm{~ms}$ (3 STD);

- independent estimation and correction cycles in Kalman filter, which allows for the prediction of the INS accuracy during unavailability of GNSS and other correctors;

- custom triple redundant processing unit for high reliability.

These novel features differentiate the developed AUV from other previously described and commercially available models [2-5].

However, IMU unit calibration was not thoroughly investigated and described, which can be considered the main limitation of this study. IMU errors are predicted in real time by Kalman filter, but performance of the IMU can be substantially improved by implementing a special calibrating procedure, which will take into account axis misalignment, temperature drifts and other errors.

\section{Conclusions}

In the course of development, the AUV's hardware and software for navigation and motion control systems were developed and implemented. Simulation results have proven operability of the developed algorithms. AUV tests made it possible to adjust synthesized algorithms as well as to re-evaluate the parameters of the AUV's analytic model. 
Future research will be focused on improving SINS calibration technics as well as developing algorithms which will allow to use sidescan sonar to correct SINS errors during underwater movement.

The reported study was financially supported by the Ministry of Science and Higher Education of the Russian Federation (Task No. 8.2118.2017/4.6).

\section{References}

[1] State standard GOST R 56960-2016 (2016). Unmanned underwater vehicles. Classification. Available at: http://docs.cntd.ru/ document/1200136057

[2] Caffaz, Turetta, Potter, Casalino, Munafo, Tay et. al. (2012). The enhanced Folaga: A hybrid AUV with modular payloads. Further Advances in Unmanned Marine Vehicles, 309-330. doi: https://doi.org/10.1049/pbce077e_ch14

[3] Eichhorn, M., Ament, C., Jacobi, M., Pfuetzenreuter, T., Karimanzira, D., Bley, K. et. al. (2018). Modular AUV System with Integrated Real-Time Water Quality Analysis. Sensors, 18 (6), 1837. doi: https://doi.org/10.3390/s18061837

[4] Zhang, M., Xu, Y., Li, B., Wang, D., Xu, W. (2014). A modular autonomous underwater vehicle for environmental sampling: System design and preliminary experimental results. OCEANS 2014 - TAIPEI. doi: https://oi.org/10.1109/oceanstaipei.2014.6964495

[5] Allotta, B., Baines, S., Bartolini, F., Bellavia, F., Colombo, C., Conti, R. et. al. (2015). Design of a modular Autonomous Underwater Vehicle for archaeological investigations. OCEANS 2015 - Genova. doi: https://oi.org/10.1109/oceans-genova.2015.7271398

[6] CAN Specification. Version 2.0 (1991). Robert Bosch GmbH. Available at: http://esd.cs.ucr.edu/webres/can20.pdf

[7] Zharkov, M. V., Veremeenko, K. K., Antonov, D. A., Kuznetsov, I. M. (2018). Attitude Determination Using Ambiguous GNSS Phase Measurements and Absolute Angular Rate Measurements. Gyroscopy and Navigation, 9 (4), 277-286. doi: https:// doi.org/10.1134/s2075108718040090

[8] Antonov, D. A., Veremeenko, K. K., Zharkov, M. V., Zimin, R. Y., Kuznetsov, I. M., Pron'kin, A. N. (2016). Test complex for the onboard navigation system of an airport ground vehicle. Journal of Computer and Systems Sciences International, 55 (5), 832-841. doi: https://doi.org/10.1134/s106423071604002x

[9] Lukomsky, Yu. A., Chugunov, V. S. (1988). Marine moving objects control systems. Leningrad: Shipbuilding.

[10] Grumondz, V. T., Polovinkin, V. V., Yakovlev, G. A. (2012). The theory of the movement of bicentric devices: mathematical models and research methods. Moscow: University book. 